\title{
La perseverancia y pasión en la consecución de objetivos: Validación española de la Escala Grit de Duckworth \\ Perseverance and passion in achieving the objectives: Spanish Validation of the Duckworth's Grit Scale
}

\author{
Maria Isabel Barriopedro, Iñaki Quintana y Luis Miguel Ruiz \\ Universidad Politécnica de Madrid. España
}

\begin{abstract}
Resumen
El concepto de Grit, que ha sido definido como perseverancia en el esfuerzo y consistencia en los intereses para la consecución de unos objetivos, ha recibido una gran atención por sus posibilidades de predecir el éxito en diferentes ámbitos. El objetivo del presente estudio fue validar la escala Grit al idioma español. Se ha contado con una muestra de 303 participantes, 123 hombres y 180 mujeres, de edades comprendidas entre los 20 y 30 años. Se comprobó mediante análisis factorial confirmatorio que la estructura de dos factores de la escala original presenta un ajuste razonable en la muestra española, tanto para los hombres como para las mujeres. Los coeficientes de fiabilidad obtenidos fueron semejantes a los de la versión original. Los factores de la escala se relacionaron positivamente con la Escala de Persistencia Motivacional. En conclusión, la versión española de la Escala Grit mantiene la estructura factorial de la original, y tiene unas propiedades psicométricas aceptables. Por lo tanto, puede resultar aplicable y útil para su empleo en diferentes entornos culturales y deportivos de habla hispana.
\end{abstract}

Palabras Clave: Persistencia; rendimiento; personalidad; medición.

\begin{abstract}
Grit, defined as perseverance of effort and consistency of interest, has attracted great attention as a predictor of success in different fields. The aim of the present study was to validate the Grit scale into the Spanish language. The sample was composed of 303 participants (123 men and 180 women) aged between 20 to 30 years. The confirmatory factor analysis showed that the two-factor structure of the original scale has a reasonable fit in the Spanish sample, in both men and women. The reliability coefficients obtained were closer to those of the original version. Additionally, the subscales were positively related to the Motivational Persistence Scale. In conclusion, the Spanish version of the Grit Scale maintains the factorial structure of the original scale and has similar psychometric properties. Therefore, it can be applicable and useful in Spanish-speaking contexts.
\end{abstract}

Key words: Persistence; achievement; personality; measurement.

Correspondencia/correspondence: Maria Isabel Barriopedro

Universidad Politécnica de Madrid. España

Email: misabel.barriopedro@upm.es 
Barriopedro, M. I.; Quintana, I., y Ruiz, L. M. (2018). La perseverancia y pasión en la consecución de objetivos: Validación española de la Escala Grit de Duckworth. RICYDE. Revista internacional de ciencias del deporte. 54(14), 297-308. https://doi.org/10.5232/ricyde2018.05401

\section{Introducción}

$\mathrm{H}$ ay hazañas que pasan a la historia por su especial relevancia. Rafael Nadal es ejemplo de deportista que ha alcanzado logros excepcionales después de haber pasado lesiones y dificultades que a cualquier otro deportista le hubiera llevado a plantearse el abandono. Tanto en su comportamiento como en sus declaraciones, este deportista ha dejado claro que la pasión, el compromiso y la determinación son claves para superar las dificultades y alcanzar los objetivos que se planteaba.

Los estudios sobre rendimiento humano vienen planteándose de forma especial de qué forma las variables no cognitivas influyen en dicho rendimiento (Cox, 1926; Terman, 1925; Hough, 1992). La investigación está mostrando las relaciones existentes entre este tipo de variables y el rendimiento académico, laboral o deportivo (Richardson, Abraham y Bond, 2012; Robbins, Lauver, Le, Langley y Carlstrom, 2004). Variables tales como la pasión (Lisbona, Palaci y Castaño, 2017; Vallerand, 2012), la inteligencia emocional (García, Ruiz y Graupera, 2013), autocontrol (Duckworth y Groos, 2014) o el compromiso (Scanlan, Carpenter, Schmidt, GSimons y Keeler (1993) han sido planteadas como variables a considerar.

En 2007 Duckworth (Duckworth, Peterson, Matthews y Kelly, 2007) propuso una noción no cognitiva que consideraba poseía el potencial de predecir el rendimiento y que denominó Grit. Esta característica personal explicaría por qué algunos individuos muestran un comportamiento especial y son capaces de incrementar su rendimiento a pesar de todas las adversidades que puedan encontrar. Para autores como Schmidt, Fleckenstein, Retelsdorf, Eskreis-Winkler y Möller (2017) este concepto haría referencia al esfuerzo desplegado por un individuo para persistir en el empeño para conseguir un objetivo en la vida, y a la consistencia en los intereses que un individuo tiene cuando los trata de alcanzar.

Para Duckworth mostrar Grit supone poseer el aguante suficiente, es vivir la vida como una maratón y no como una carrera de velocidad (Duckworth, 2013; Duckworth y col., 2007). En sus propias palabras supone" trabajar intensamente ante los retos, mantener el esfuerzo $e$ interés a lo largo de los años a pesar de la adversidad, error, y mesetas en el progreso" (Duckworth y col., 2007, pp. 1087-1088).

Existe numerosa evidencia anecdótica, así como información biográfica de grandes personajes caracterizados por haber alcanzado grandes logros (Galton, 1892; Terman, 1925) que muestran que el empeño, la inversión personal, el compromiso, la dedicación plena y el esfuerzo sostenido durante años, son elementos imprescindibles a la hora de explicar su éxito en diferentes ámbitos de actuación (empresa, milicia, deporte, medicina, ciencia, etc.).

Los estudios de Ericsson sobre la práctica deliberada y el entrenamiento intenso abundan en esta dirección (Duckworth, Kirby, Tsukayama, Bernstein y Ericsson, 2011; Ericsson, 2017; Ericsson, Krampe y Tesch-Römer, 1993), ya que para este investigador ser capaz de estar miles de horas trabajando, o entrenando, en la consecución de retos y objetivos, supone poseer el Grit necesario para no abandonar y persistir a pesar de las dificultades. Es por ello por lo que se empieza a plantear si solo el talento y la inteligencia por sí mismos debieran ser considerados los únicos causantes del éxito académico, científico, empresarial o deportivo (Van Rossum y Gagne, 2005).

Duckworth et al. (2007) desarrollaron una escala de autoinforme compuesta de 12 ítems en las que ambas dimensiones estaban presentes y que denominó Escala Grit-12. Estas variables la concretaron en dos dimensiones: Perseverancia en el esfuerzo y Consistencia en los intereses. La consistencia en los intereses haría referencia a la tendencia a no cambiar de intereses de manera habitual o frecuente, mientras que perseverancia en el esfuerzo indicaría la tendencia a 
Barriopedro, M. I.; Quintana, I., y Ruiz, L. M. (2018). La perseverancia y pasión en la consecución de objetivos: Validación española de la Escala Grit de Duckworth. RICYDE. Revista internacional de ciencias del deporte. 54(14), 297-308. https://doi.org/10.5232/ricyde2018.05401

trabajar duro incluso en las situaciones más difíciles durante mucho tiempo a pesar de las inconveniencias y dificultades. Estos autores analizaron la validez incremental del Grit en contraposición a la inteligencia en lugares como la academia militar norteamericana de West Point, el Campeonato nacional de deletreo (National Spelling Bee) y la valoración promedio en la institución Ivy League, encontrando que aquellos participantes que puntuaban más alto en la escala tenían la tendencia a trabajar más duro y durante más tiempo que sus compañeros menos gritty, y que esta dimensión podría ser la causa de sus diferencias de rendimiento, e incluso frente al error. En 2009 Duckworth y Quinn revisaron la escala propuesta inicialmente y encontraron que la eliminación de algunos de sus ítems (dos de cada dimensión) mejoraba notablemente la fortaleza de su modelo, quedando establecida la escala como Escala GRIT-S versión reducida de 8 ítems, compuesta por dos dimensiones y un factor superior denominado Grit.

Hasta la fecha esta escala ha sido validada en algunos países europeos (Dumfart y Neubauer, 2016; Schmidt y col., 2017), no existiendo ningún estudio de validación publicado en el contexto español, de ahí la importancia del presente estudio para la Psicología del Deporte y las Ciencias del Deporte dada la existencia de numerosos instrumentos que no han sido sometidos a la necesaria y correspondiente validación y adaptación cultural. Es por ello por lo que el presente estudio tiene como propósito la validación en población española de la escala de pasión y Perseverancia en la consecución de objetivos GRIT, tanto en su versión original (GRIT-O) como en su versión reducida (GRIT-S).

\section{Método}

\section{Participantes}

Fueron 303 personas las que participaron en este estudio. La muestra fue de carácter no probabilístico. Las edades estuvieron comprendidas entre los 20 y los 30 años, de los que 123 eran varones $(M=20.93$; $D T=2.6$ años) y 180 mujeres $(M=20.99$; $D T=3.1$ años $)$. El 75.4\% fueron estudiantes universitarios en centros superiores de Ciencias del Deporte de sus diferentes cursos, y el $24.6 \%$ fueron deportistas de diferentes modalidades y niveles. Todos ellos participaron de manera voluntaria

\section{Instrumentos}

\section{Escala Grit-Original (Grit-O)}

Para llevar a cabo esta validación los participantes cumplimentaron de forma voluntaria la escala Grit original (Grit-O) de 12 ítems (Duckworth y col., 2007) traducida al español. Para la traducción del cuestionario se tuvieron en cuenta las recomendaciones para la adaptación de los tests de unas culturas a otras (Muñiz y Hambleton, 1996). Un traductor bilingüe, con conocimientos sobre los contenidos específicos del presente trabajo y sobre los principios de la construcción de tests, tradujo la totalidad de los ítems de la versión en inglés del Grit-O. Un segundo traductor bilingüe tradujo la versión en castellano de nuevo al inglés. Finalmente, dos investigadores expertos en el área compararon esta traducción con el cuestionario original en inglés. Por último, los traductores y los investigadores consensuaron una versión final.

El cuestionario Grit-O estuvo formado por dos factores: consistencia (integrada por los ítems $2,3,5,7,8$ y 11) y perseverancia (integrada por los ítems 1, 4, 6, 9, 10 y 12) (véase Anexo). Duckworth y Quinn (2009) propusieron una versión reducida de este cuestionario denominada Grit-S, en el que eliminaron los ítems 3 y 11 de la dimensión consistencia y los ítems 1 y 10 de la de perseverancia. Se mantuvo el formato de respuesta del instrumento original siendo $5=$ Muy parecido a mí a $1=$ En absoluto se parece a mí. 
Barriopedro, M. I.; Quintana, I., y Ruiz, L. M. (2018). La perseverancia y pasión en la consecución de objetivos: Validación española de la Escala Grit de Duckworth. RICYDE. Revista internacional de ciencias del deporte. 54(14), 297-308. https://doi.org/10.5232/ricyde2018.05401

Junto con la escala Grit-O, los participantes también cumplimentaron la Escala de Persistencia Motivacional de Constantin, Holman y Hojbota (2011) para establecer su validez convergente. Esta escala consta de 13 ítems y evalúa tres dimensiones: a) Consecución de objetivos a corto plazo (4 ítems), b) Consecución de objetivos a largo plazo (4 ítems) y c) Vuelta a objetivos no conseguidos (5 ítems). Por consecución de objetivos concretos a corto plazo los autores entienden la capacidad de mantenerse centrado y de mantener el esfuerzo por alcanzar logros concretos a pesar del aburrimiento, la fatiga o el estrés. Por perseguir objetivos a largo plazo resaltan mantenerse firme y persistir en la consecución de objetivos en un plazo de tiempo más largo. Por último, por volver a objetivos anteriores, los autores destacan ser capaces de restaurar el compromiso que una vez se tuvo para alcanzar un reto. El formato de respuesta también fue de 5 puntos (1 totalmente en desacuerdo a 5 Totalmente de acuerdo).

\section{Procedimiento}

Los participantes fueron invitados a colaborar en el estudio explicándoles la finalidad del mismo. Todos firmaron una hoja consentimiento con la que iniciaba el cuadernillo de cuestionarios. Completaron, sin límite de tiempo, un cuadernillo que incluía la escala Grit-O traducido al castellano. Cada ítem de la escala fue respondido utilizando un formato de respuesta politómico con 5 alternativas: a) Muy parecido a mí, b) Bastante parecido a mí, c) Un poco como yo, d) Poco parecido conmigo y e) En absoluto se parece a mí. Estas opciones de repuesta fueron valoradas de 1 a 5 , respectivamente, para los ítems $2,3,5,7,8$ y 11 , y con puntuaciones de 5 a 1 para el resto de los ítems. Una submuestra de 74 participantes cumplimentó también la Escala de Persistencia Motivacional de Constantin, Holman y Hojbota (2011) en su versión española. Los ítems de la escala de Persistencia Motivacional, el sistema de respuesta fue una escala tipo Likert de 1 (totalmente en desacuerdo) a 5 (totalmente de acuerdo).

En todo momento se tuvo en cuenta las consideraciones éticas necesarias para el desarrollo de este tipo de estudios (https://www.cop.es/index.php?page=principios-eticos).

\section{Análisis Estadístico}

La estructura factorial propuesta por Duckworth y col. (2007) tanto para la versión original (Grit-O) y su versión reducida (Grit-S) propuesta por Duckworth y Quinn (2009), fueron sometidas a análisis factoriales confirmatorios utilizando el método de Máxima Verosimilitud para estimar los parámetros, tras comprobar que tanto los índices de Asimetría como los de Apuntamiento de los ítems no presentaban valores elevados (Asimetría menor que 2 en valor absoluto; Apuntamiento menor que 7 en valor absoluto). Para evaluar la bondad de ajuste de los datos al modelo propuesto se utilizaron los siguientes índices: Raíz del Error Cuadrático Medio de Aproximación (RMSEA) y su intervalo de confianza al 90\%, Índice de Ajuste Comparado de Bentler (CFI), Índice de Bondad de Ajuste (GFI) y el valor relativo de Chi Cuadrado $\left(\chi^{2} / \mathrm{gl}\right)$.

En la literatura, valores de RMSEA $\leq, 05$ o ,08 se consideran como indicadores de un buen ajuste o un ajuste aceptable, respectivamente; valores de CFI $\geq, 90$ y de GFI $\geq, 95$ han sido aceptados como indicadores de un buen ajuste; por último, valores relativos de Chi Cuadrado entre 2 y 5 se consideran como indicadores de ajuste aceptable. Para comprobar si la estructura factorial era igual en hombres y mujeres se realizaron AFC multigrupo. La invarianza factorial se evaluó de forma progresiva (Byrne, 2008): invarianza configural (el patrón de cargas factoriales es el mismo); invarianza métrica o débil (además del patrón de cargas, los pesos factoriales son iguales); invarianza escalar o fuerte (además de invarianza métrica supone que los interceptos son iguales) e invarianza estricta (además de la invarianza escalar supone varianzas iguales para los errores). 
Dado que la diferencia entre los test de Chi-Cuadrado es muy restrictiva, para comparar el ajuste de los modelos se utilizó además el Criterio de Información de Akaike (AIC) y la comparación de los valores del CFI. Si la diferencia entre los valores CFI de dos modelos anidados es superior a .01 a favor del modelo menos restrictivo deberá rechazarse el modelo con más restricciones (Chenung y Rensvold, 2002). La fiabilidad de las escalas se evaluó mediante el coeficiente $\alpha$ y la homogeneidad de los ítems a partir de la correlación entre el ítem y la puntuación total de la escala una vez eliminado el ítem. Los análisis fueron realizados mediante el programa PASW 20.0 y el programa AMOS 21.0.

\section{Resultados}

\section{Dimensionalidad y Fiabilidad de la escala GRIT}

El ajuste de la estructura de dos factores para la escala original (Grit-O) mostró un ajuste pobre: $\mathrm{RMSEA}=, 120 ; \mathrm{CFI}=, 87 ; \mathrm{GFI}=, 88 ; \chi^{2} / 54=5,38$. La estructura de dos factores para la versión reducida, Grit-S, arrojó un ajuste similar al informado por los autores: RMSEA =,041 (,001,070); CFI =,96; GFI = ,98; $\chi^{2} / 19=1,51$. La consistencia interna, de la dimensión Consistencia en los intereses fue de ,68 y la de Perseverancia .54. En esta última dimensión la consistencia mejoró hasta ,64 al eliminar el ítem 4. La consistencia total de la escala fue de, .66. Las correlaciones de los ítems con el total de la escala y con el total del factor eliminando el ítem, presentaron valores entre ,31 y ,56 para el factor Consistencia, y de entre ,39 y ,40 para la Perseverancia. No obstante, el ítem 4 de esta escala presentó una baja correlación con el total de la escala $(, 16)$.

Los ítems fueron buenos indicadores de sus respectivos factores latentes, con saturaciones factoriales estandariazadas entre 0,38 y 0,74 para la subescala de Consistencia en los intereses y entre, 41 y ,69 para la subescala de Persistencia, excepto para el ítem 4, que presentó una saturación de ,09. Los factores consistencia y perseverancia mostraron saturaciones de ,92 y ,55, respectivamente, en el factor de segundo orden. Todas las saturaciones resultaron estadísticamente significativas $(p<, 001)$ excepto la del ítem $4(p=, 193)$ (Fig. 1).

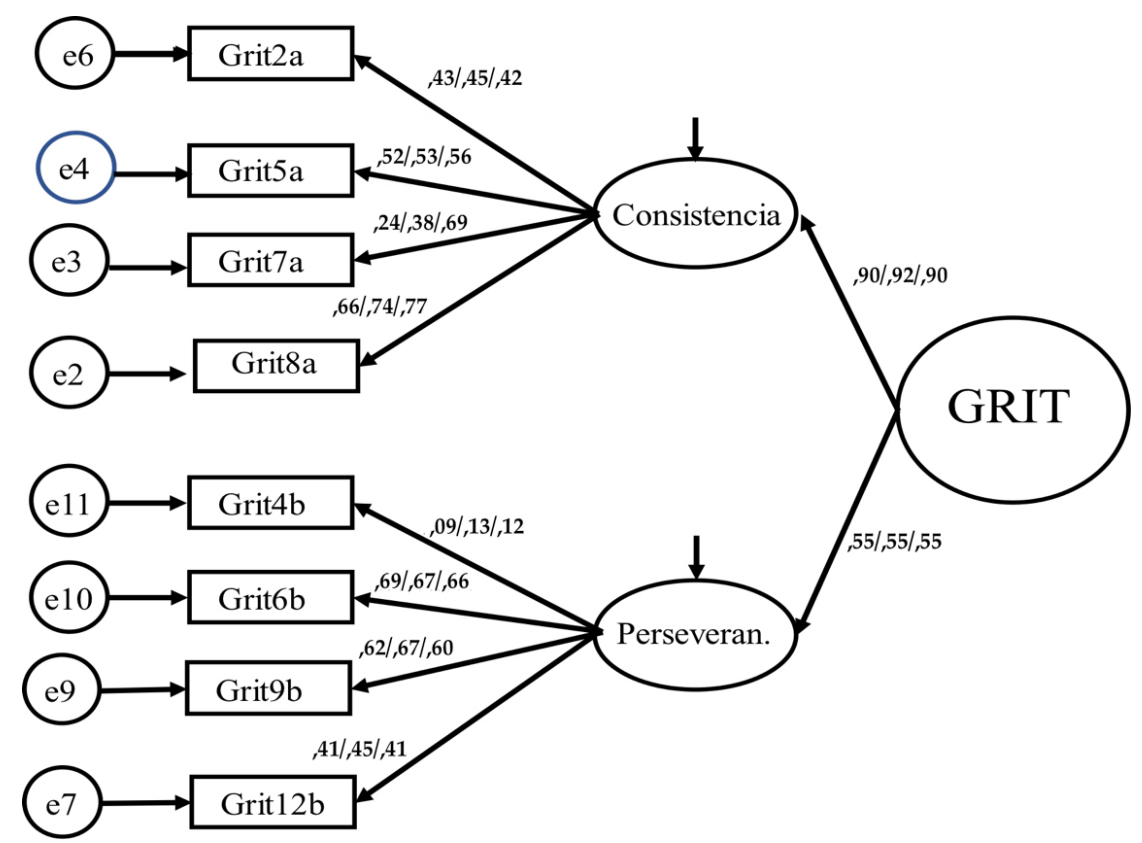

Fig. 1. Estructura factorial del Cuestionario Grit-O con sus saturaciones factoriales estandarizadas para todo el cuestionario, hombres y mujeres. 
Barriopedro, M. I.; Quintana, I., y Ruiz, L. M. (2018). La perseverancia y pasión en la consecución de objetivos: Validación española de la Escala Grit de Duckworth. RICYDE. Revista internacional de ciencias del deporte. 54(14), 297-308. https://doi.org/10.5232/ricyde2018.05401

\section{Validez convergente}

Para evaluar la validez convergente de la escala Grit-S se utilizó la Escala de Persistencia Motivacional. El factor Consistencia en los intereses mostró correlaciones significativas con las subescalas Consecución de objetivos a corto plazo (COCP) $(\mathrm{r}=, 50)$ y a largo plazo (COLP) $(\mathrm{r}=, 52)$ y una relación inversa con la subescala Vuelta a los objetivos no conseguidos $(V O)(\mathrm{r}$ $=-, 28)$. El factor Perseverancia mostró correlaciones significativas con las subescalas Consecución de objetivos a corto plazo $(\mathrm{r}=, 64)$ y a largo plazo $(\mathrm{r}=-, 54)$. (Tabla 1).

Tabla 1. Relaciones entre los factores de la Escala Grit-O y las dimensiones de la Escala de Persistencia Motivacional.

\begin{tabular}{lcll}
\hline & VO & COCP & COLP \\
\hline Consistencia &,$- 281^{*}$ &, $503^{* *}$ &, $521^{* *}$ \\
Perseverancia &,- 079 &, $648^{* *}$ &, $5431^{* *}$ \\
\hline
\end{tabular}

Invarianza Factorial

La escala mostró índices de ajuste aceptables (Tabla 2) tanto para los varones (RMSEA $=, 058$ y $\mathrm{CFI}=, 95)$ como para las mujeres $(\mathrm{RMSEA}=, 048$ y $\mathrm{CFI}=, 96)$. Los índices obtenidos permiten aceptar la equivalencia del patrón de cargas factoriales (invarianza configural) para ambos grupos de personas (RMSEA = ,037 y CFI = ,973). Cuando al modelo se le añadieron restricciones sobre los pesos factoriales (invarianza métrica) se obtuvo un mal ajuste del modelo $(\mathrm{RMSEA}=, 073$ y $\mathrm{CFI}=, 872)$, sufriendo el índice comparativo de Bentler una reducción mayor de 0,1 y el criterio de información de Akaike $(\mathrm{AIC}=204,8)$ una modificación considerable. Estos resultados no permiten aceptar la invarianza métrica.

Tabla 2. Índices de ajuste de la escala Grit-O

\begin{tabular}{lcccccc}
\hline Invarianza & $\chi^{2}$ & gl & $\chi^{2} /$ gl & RMSEA (IC al 90\%) & CFI & AIC \\
\hline Hombres & 26,7 & 19 & 1,40 &, $058(, 000-, 105)$ &, 946 & 60,7 \\
Mujeres & 27,0 & 19 & 1,42 &, $048(, 000-, 087)$ &, 964 & 61,0 \\
Configural & 55,2 & 39 & 1,41 &, $037(, 006-, 058)$ &, 973 & 153,2 \\
Métrica & 122,8 & 47 & 2,62 &, $073(, 057-, 089)$ &, 872 & 204,8 \\
\hline
\end{tabular}

\section{Discusión}

La escala Grit se ha convertido en un instrumento cuyo empleo es amplio en el ámbito norteamericano y que ha recibido la atención de numerosos investigadores europeos, traduciéndose y adaptándose a varios idiomas (ver la página de la propia autora, https://sites.sas.ipenn.edu/duckworth/pages/research) así como sus publicaciones (Duckworth, 2016). El presente estudio es el primero en el que se realiza una validación al español del cuestionario Grit (Duckworth y col., 2007). Este instrumento posee dos dimensiones denominadas Consistencia y Persistencia, aunque en los estudios lo que habitualmente se suele ofrecer son los valores globales de la escala, ya que como indica la autora ninguno de ellos por separado ofrece un mayor poder predictivo de éxito, siendo los dos los que mejor representan el constructo estudiado (Duckworth y col., 2007). 
Barriopedro, M. I.; Quintana, I., y Ruiz, L. M. (2018). La perseverancia y pasión en la consecución de objetivos: Validación española de la Escala Grit de Duckworth. RICYDE. Revista internacional de ciencias del deporte. 54(14), 297-308. https://doi.org/10.5232/ricyde2018.05401

El análisis de la validez factorial de las puntuaciones de la adaptación de la escala, mediante análisis factorial confirmatorio, arrojó un ajuste pobre de la estructura de dos factores con un factor de segundo orden. Estos resultados son congruentes con los informados por Duckworth y col. (2007) quienes tampoco mostraron un buen ajuste para su modelo (i.e. $\mathrm{CFI}=, 83$, RMSEA $=, 11)$. En su versión reducida (Grit-S), la validez de la escala se ajusta de forma satisfactoria a los datos, mejorando los niveles de ajuste obtenidos en diversas muestras por Duckworth y Quinn (2009).

En cuanto a la fiabilidad, las puntuaciones en la escala original Grit-O mostraron una aceptable consistencia interna, tanto para los dos factores como para la escala total, similares a los informados por Duckworth y Quinn (2009). Además, como se esperaba, las puntuaciones de los factores Consistencia en los intereses y Perseverancia en el esfuerzo correlacionaron significativamente con las dimensiones Consecución de objetivos a corto y largo plazo de la Escala de Persistencia Motivacional, mostrando una adecuada validez discriminante. Estas relaciones son similares a las obtenidas por Constatin y col. (2011), quienes hallaron relaciones moderadas entre las dos dimensiones del Grit y las dimensiones de la Escala de Persistencia Motivacional.

Schmidt y col. (2017) encontraron validez discriminante y convergente de la escala GRIT-S al relacionarla con diferentes instrumentos de autoinforme relacionados con la autoeficacia, autocontrol o escrupulosidad. El estudio progresivo de la invarianza factorial entre varones y mujeres mostró un ajuste adecuado del modelo lo que supone una invarianza parcial, es decir, aquella que implica que además del patrón de cargas factoriales, los pesos factoriales son iguales para ambos grupos. Sólo el ítem 7 presentó cargas factoriales significativamente diferentes en varones y mujeres. Esto indica que, aunque éste ítem se relaciona con el mismo constructo en varones y mujeres, lo hace con un grado diferente de asociación.

Por otro lado, ninguno de los modelos de invarianza escalar (total o parcial) presentó ajustes aceptables. Estos resultados no son congruentes con los informados por Schmidt y col. (2017), quienes proponen una invarianza escalar parcial para su adaptación al alemán de esta misma escala, pero sobre una muestra de mayor edad a la utilizada en este estudio. Algunos autores sostienen la necesidad de comprobar la igualdad de los interceptos para poder comparar las medias de los factores entre grupos, aunque sería suficiente con una invarianza escalar parcial, es decir, bastaría que se cumpliese para una parte de los ítems de cada factor (Hui y Triandis, 1985).

\section{Limitaciones y prospectiva de futuro}

Se deben destacar algunas limitaciones del presente estudio. En primer lugar, la muestra está formada preferentemente por personas relacionadas con el ámbito del deporte, bien por ser universitarios de Facultades de Ciencias de la Actividad Física y el Deporte, o porque eran deportistas en activo de diferente nivel de pericia. Esto implica que hace difícil la generalización de los resultados al resto de la población. En segundo lugar, parece necesario una ampliación de las muestras a contextos de logro en los que la persecución de objetivos, el manteniendo del esfuerzo e interés a lo largo del tiempo y la consistencia en los objetivos, sea muy característico. Ámbitos como el deporte de alto rendimiento, la empresa, el mundo académico o el militar, parecen ámbitos adecuados para seguir estudiando este constructo en el contexto español. Cuarto, frente al estudio de tipo transversal que se ha presentado en este artículo, parece adecuado realizar estudios de carácter longitudinal, en los que se pueda ver la estabilidad o el cambio del constructo al largo de los años. 
Barriopedro, M. I.; Quintana, I., y Ruiz, L. M. (2018). La perseverancia y pasión en la consecución de objetivos: Validación española de la Escala Grit de Duckworth. RICYDE. Revista internacional de ciencias del deporte. 54(14), 297-308. https://doi.org/10.5232/ricyde2018.05401

Como quinta limitación destacar los problemas que en muchas ocasiones presentan los instrumentos de autoinforme, como muy bien la propia autora de la escala dejó patente (Duckworth y Yager, 2015), y los problemas derivados de la deseabilidad social. En resumen, aunque el cuestionario Grit-S muestra propiedades psicométricas más adecuadas que la versión original, el Grit-O, se hace necesario seguir profundizando sobre la medición de este constructo para la obtención de un instrumento que permita comparar las puntuaciones de grupos de participantes con diferentes características.

Por último, destacar que el meta-análisis realizado por Crede, Tyann y Harms (2016) ha destacado algunos aspectos de este constructo, y de su investigación, que necesitan una mayor atención, como el ámbito en el que se aplica, el nivel de pericia de los participantes o incluso la definición del propio constructo que presenta similitudes con otros constructos ya existentes como el de autodisciplina que Costa y McCrae (1992) definen como la capacidad para iniciar y terminar tareas a pesar del aburrimiento o las distracciones, de manera esforzada y concienzuda. Para Crede, Tyann y Harms (2016) la noción de grit es intuitivamente atrayente cuando se la considera como predictora del rendimiento, pero los datos no apoyan de forma tan rotunda esta capacidad predictiva.

El presente estudio permite que los investigadores de las Ciencias del Deporte de habla hispana dispongan, con confianza, de un cuestionario que permita analizar la perseverancia y pasión de los individuos en la consecución de sus objetivos, y favorecer la investigación en este ámbito. Para los entrenadores, instructores y técnicos y se ofrece un instrumento, de sencilla y fácil aplicación, con todas las cualidades de medida necesarias y exigibles, que les puede dotar de información muy valiosa sobre la personalidad perseverante o gritty de sus entrenados.

\section{Referencias}

Ali, J., \& Rahaman, A. (2012). A comparative study of grit between male and female fencers of Manipur. International Journal of Movement Education and Social Sciences, 2(1), 10-12.

Buller, E. F. (2012). The Relationship between Grit and Academic, Military and Physical Performance at the United States Military Academy. (Doctoral Dissertation). University of Kansas, Kansas (USA).

Byrne, B. M. (2008). Testing for multigroup equivalence of a measuring instrument: A walk through the process. Psicothema, 20(4), 872-882

Chenung, G. W., \& Rensvold, R.B. (2002). Evaluating Goodness-of-Fit Indexes for Testing Measurement Invariance. Structural Equation Modeling, 9(2), 233-255

Constantin, T.; Holman, A., \& Hojbota, M. A. (2011). Development and Validation of a Motivational Persistence Scale. Psihologija, 45 (2), 99-120. https://doi.org/10.2298/PSI1202099C

Costa, P.T. Jr., \& McCrae, R. R. (1992). The Revised NEO Personality Inventory (NEO-PI$R$ ) and NEO-Five-Factor Inventory (NEO-FFI) Professional Manual. Odessa, FL: Psychological Assessment Resources.

Cox, C. M. (1926). The Early Mental Traits of Three Hundred Geniuses. Stanford, CA: Stanford University Press.

Credé, M.; Tynan, M. C., \& Harms, P. D. (2017). Much a do about Grit: A meta-analytic synthesis of Grit literature. Journal of Personality and Social Psychology 113(3), 492511. https://doi.org/ 10.1037/pspp0000102 
Datu, J. A. D.; Yuen, M., \& Chen, G. (2016). Exploring determination for long-term goals in a collectivist context: A qualitative study. Current Psychology, 1-9. https://doi.org/10.1017/jgc.2016.2

Duckworth, A (2013). Grit: the power of passion and perseverance. Recuperado de: https://www.youtube.com/watch?v=H14bBuluwB8

Duckworth, A. (2016). Grit. El poder de la pasión y la perseverancia. Madrid: Ediciones Urano.

Duckworth, A. L.; Peterson, C.; Matthews, M. D., \& Kelly, D. R. (2007). Grit: Perseverance and passion for long-term goals. Journal of personality and social psychology, 92(6), 1087-1101.

Duckworth, A. L., \& Quinn, P. D. (2009). Development and validation of the short grit scale (grit-s). Journal of personality assessment, 91(2), 166-174.

https://doi.org/10.1080/00223890802634290

Duckworth, A. L.; Kirby, T. A.; Tsukayama, E.; Berstein, H., \& Ericsson, K. A. (2011). Deliberate practice spells success why grittier competitors triumph at the national spelling bee. Social Psychological and Personality Science, 2(2), 174-181. http://dx.doi.org/10.1177/1948550610385872

Duckworth, A. L.; Quinn, P. D.; Lynam, D. R.; Loeber, R., \& Stouthamer-Loeber, M. (2011). Role of test motivation in intelligence testing. Proceedings of the National Academy of Sciences, 108(19), 7716-7720.

https://doi.org/10.1073/pnas.1018601108

Duckworth, A., \& Groos, J. J. (2014). Self-Control and Grit: Related but Separable Determinants of Success. Current Directions in Psychological Science., 23(5), 319-325. https://doi.org/10.1177/0963721414541462

Duckworth, A., \& Yager, D. S. (2015). Measurement Matters Assessing Personal Qualities Other Than Cognitive Ability for Educational Purposes. Educational Researcher, 44(4), 237-251. https://doi.org/10.3102/0013189X15584327

Dumfart, B., \& Neubauer, A. C. (2016). Conscientiousness Is the Most Powerful Noncognitive Predictor of School Achievement in Adolescents. Journal of Individual Differences, 37(1), 8-15. https://doi.org/10.1027/1614-0001/a000182

Ericsson, K. A.; Krampe, R. T., \& Tesch-Römer, C. (1993). The role of deliberate practice in the acquisition of expert performance. Psychological Review, 100(3), 363-406.

Ericsson, K. A., \& Charness, N. (1994). Expert performance: Its structure and acquisition. American Psychologist, 49(8), 725-747.

http://dx.doi.org/10.1037/0003-066X.49.8.725

Eskreis-Winkler, L., Shulman, E. P., Beal, S. A., \& Duckworth, A. L. (2014). The Grit effect: Predicting retention in the military, the workplace, school and marriage. Frontiers in Psychology, 5 (36), 1-12.

https://doi.org/10.3389/fpsyg.2014.00036

Galton, F. (1892). Finger Prints. Londres: Mcmillan

García, V., Ruiz, L. M., \& Graupera, J. L. (2013). Inteligencia Emocional en el Deporte: Validación española del Schutte Self Report Inventory (SSRI) en deportistas españoles. Cuadernos de Psicología del Deporte, 13(1), 25-36.

Hui, C. H., \& Triandis, H.C. (1985) Measurement in Cross-Cultural Psychology: A review and comparison of strategies. Journal of Cross-Cultural Psychology, 16(2): 131-152. https://doi.org/10.1177/0022002185016002001 
Barriopedro, M. I.; Quintana, I., y Ruiz, L. M. (2018). La perseverancia y pasión en la consecución de objetivos: Validación española de la Escala Grit de Duckworth. RICYDE. Revista internacional de ciencias del deporte. 54(14), 297-308. https://doi.org/10.5232/ricyde2018.05401

Hough, L. M. (1992). The 'big five' personality variables construct confusion: Description versus prediction. Human performance, 5(1-2), 139-155.

John, O. P., \& Srivastava, S. (1999). The big five trait taxonomy: History, measurement, and theoretical perspectives. Handbook of personality: Theory and research, 2(1999), 102-138.

Kim, Y-J. (2015). The International Comparison on the Grit and Achievement Goal Orientation of College Students: Focusing on the college students in Korea, China, and Japan. Advanced Science and Technology Letters, 119, 10-13.

Lievens, F., \& Sackett, P. R. (2012). The validity of interpersonal skills assessment via situational judgment tests for predicting academic success and job performance. Journal of Applied Psychology, 97(2), 460-468. https://doi.org/10.1037/a0025741

Lisbona, A.; Palací, F. J., y Castaño, M. B. (2016). ¿Podemos apasionarnos por el trabajo?: Una revisión de la pasión para el trabajo. Papeles del Psicólogo, 37 (3), 165-169. http://www.papelesdelpsicologo.es/pdf/1172.pdf

Muñiz, J., y Hambleton, R. K. (1996). Directrices para la traducción y adaptación de los tests. Papeles del Psicólogo, 66.

http://www.papelesdelpsicologo.es/resumen?pii=737

Rimfeld, K.; Kovas, Y.; Dale, P. S., \& Plomin, R. (2016). True grit and genetics: Predicting academic achievement from personality. Journal of Personality and Social Psychology, 111(5), 780-789. https://doi.org/10.1037/pspp0000089

Richardson, M.; Abraham, C., \& Bond,R. (2012). Psychological correlates of university students' academic performance: a systematic review and meta-analysis. Psychological Bulletin, 138(2).

https://doi.org/10.103/a0026838

Robbins, S. B.; Lauver, K.; Le, H. ; Davis, D.; Langley, R., \& Carlstrom, A. (2004). Do psychosocial and study skill factors predict college outcomes? A meta-analysis. Psychological Bulletin, 130(2), 261-288. https://doi.org/ 10.1037/0033-2909.130.2.261

Robertson-Kraft, C., \& Duckworth, A. L. (2014). True grit: Trait-level perseverance and passion for long-term goals predicts effectiveness and retention among novice teachers. Teachers College Record, 116(3), 1-27.

Salles, A.; Cohen, G. L., \& Mueller, C. M. (2014). The relationship between grit and resident well-being. The American Journal of Surgery, 207(2), 251-254.

https://doi.org/ 10.1016/j.amjsurg.2013.09.006

Scanlan, T. K.; Carpenter, P. J.; Schmidt, G. W.; Simons, J. P., \& Keeler, B. (1993). An introduction to the sport commitment model. Journal of Sport \& Exercise Psychology, $15,1-15$.

Schmidt, F. T. C.; Fleckenstein, J.; Retelsdorf, J.; Eskreis-Winkler, L., \& Möller, J. (2017). Measuring Grit. A German validation and a domain-specific approach to Grit. European Journal of Psychological Assessment. https://doi.org/10.1027/1015-5759/a000407

Silvia, P. J.; Eddington, K. M.; Beaty, R. E.; Nusbaum, E. C., \& Kwapil, T. R. (2013). Gritty people try harder: Grit and effort-related cardiac autonomic activity during an active coping challenge. International Journal of Psychophysiology, 88(2), 200-205. https://doi.org/ 10.1016/j.ijpsycho.2013.04.007 
Singh, K., \& Jha, S. D. (2008). Positive and negative affect, and grit as predictors of happiness and life satisfaction. Journal of the Indian Academy of Applied Psychology, $34(2), 40-45$.

Terman, L. M. (1925). Genetic studies of genius. Mental and physical traits of a thousand gifted children. Palo Alto, CA: Stanford Univ. Press.

Vallerand, R. J. (2012). The role of passion in sustainable psychological well-being. Psychology of Well-Being: Theory, Research and Practice, 2(1). http://www.psywb.com/content/2/1/1

Van Rossum, J. H. A., \& Gagné, F. (2005) Talent development in sports. En F.A. Dixon, y S. M. Moon (eds.), The Handbook of Secondary Gifted Education (pp. 281-316). Waco, TX: Prufrock Press.

Von Culin, K. R.; Tsukayama, E., \& Duckworth, A. L. (2014). Unpacking grit: Motivational correlates of perseverance and passion for long-term goals. The Journal of Positive Psychology, 9(4), 306-312.

http://dx.doi.org/10.1080/17439760.2014.898320 


\section{Apéndice A}

\section{Ítems de la Escala Grit-O}

1. He superado contratiempos para conseguir un reto importante.

Las ideas y proyectos nuevos a menudo me distraen de los anteriores. *

3. Mis intereses cambian de año a año. *

4. Los contratiempos no me desaniman.

5. Me he obsesionado con cierta idea, o proyecto, durante un periodo corto de tiempo, para después dejar de estar interesado. *

6. Soy un trabajador/a duro/a.

7. A menudo me pongo un objetivo para después perseguir otro diferente. *

8. Tengo dificultad para mantener mi atención en proyectos que me reclaman más de varios meses llevarlos a cabo.

9. Termino todo lo que empiezo.

10. He conseguido objetivos que me costaron años alcanzarlos.

11. Me he llegado a interesar por nuevas actividades cada pocos meses. *

12. Soy una persona diligente.

\section{Puntuación:}

- Para los ítems 1, 4, 6, 9, 10 y 12 la puntuación es la siguiente:

5 = Muy parecido a mí.

$4=$ Preferentemente como yo.

$3=$ De algún modo como yo.

$2=$ No como yo.

1 = En absoluto se parece a mí.

- Para los ítems 2, 3, 5, 7, 8 y 11 la puntuación es la siguiente:

$1=$ Muy parecido a mí.

2 = Preferentemente como yo.

$3=$ De algún modo como yo.

$4=$ No como yo.

5 = En absoluto se parece a mí.

Sumar todas las puntuaciones y dividirlo entre 12. La máxima puntuación de la esca es 5 (exteremadamente gritty), y la más baja puntuación de la escala es 1 (de ninguna manera gritty). 